\title{
Predictability and Chaotic Nature of Daily Streamflow
}

\author{
D. Nagesh $\mathrm{Kumar}^{1}$, C.T. Dhanya ${ }^{1}$ \\ ${ }^{1}$ Department of Civil Engineering \\ Indian Institute of Science \\ Bangalore \\ INDIA \\ E-mail: nagesh@civil.iisc.ernet.in
}

\begin{abstract}
The predictability of a chaotic series is limited to a few future time steps due to its sensitivity to initial conditions and the exponential divergence of the trajectories. Over the years, streamflow has been considered as a stochastic system in many approaches. In this study, the chaotic nature of daily streamflow is investigated using autocorrelation function, Fourier spectrum, correlation dimension method (Grassberger-Procaccia algorithm) and false nearest neighbor method. Embedding dimensions of 6-7 obtained indicates the possible presence of low-dimensional chaotic behavior. The predictability of the system is estimated by calculating the system's Lyapunov exponent. A positive maximum Lyapunov exponent of 0.167 indicates that the system is chaotic and unstable with a maximum predictability of only 6 days. These results give a positive indication towards considering streamflow as a low dimensional chaotic system than as a stochastic system.
\end{abstract}

Keywords: Correlation dimension, Lyapunov exponent, nearest neighbour, nonlinear prediction

\section{INTRODUCTION}

The development of various climate models that numerically integrate an adequate set of mathematical equations of physical laws governing the climatic processes marked a major breakthrough in the routine weather prediction. The mathematical equations in these climate models form a nonlinear dynamical system in which an infinitesimally small uncertainty in the initial conditions will grow exponentially even under a perfect model, leading to a chaotic behavior (Smith et al, 1998). Such sensitivity of any deterministic system to a slight change in the initial conditions leads to a vast change in the final solution and is often known as "butterfly effect" in the field of weather forecasting (Lorenz, 1972). Hence, earth's weather can be treated as a chaotic system with a finite limit in the predictability, arising mainly due to the incompleteness of initial conditions. The exponential growth with time of an infinitesimal initial uncertainty $\partial_{0}$ is given by the highest Lyapunov exponent $\lambda$ (Wolf et al., 1985; Rosenstein et al., 1993). Hence, the separation or uncertainty after $\Delta t$ time steps ahead is given as $\partial_{\Delta t} \cong e^{\lambda \Delta t} \partial_{0}$. The predictability of a chaotic system is therefore limited (i) due to the indefiniteness in the initial conditions (given a perfect model) and also (ii) due to the imperfection of the model.

Modeling of many weather phenomena have been done so far employing the concept of stochastic systems. However, a large number of studies employing the science of chaos to model and predict various hydrological phenomena have emerged only in the past decade (Elshorbagy et al., 2002; Islam and Sivakumar, 2002; Jayawardena and Lai, 1994; Porporato and Ridolfi, 1996, 1997; Puente and Obregon, 1996; Rodriguez-Iturbe et al., 1989, Liu et al., 1998; Sangoyomi et al., 1996; Sivakumar et al., 1999; Sivakumar, 2001; Sivakumar et al., 2001; Shang et al., 2009; Wang and Gan, 1998). Most of these studies dealt with scalar time series data of various hydrological phenomena like rainfall, runoff, sediment transport, lake volume etc. In these cases, since neither the mathematical relations nor the influencing variables are known, the state space in which the variable is lying is reconstructed from the time series itself using phase space reconstruction method by Takens (1981). The phase space reconstruction provides a simplified, multi-dimensional representation of a single-dimensional nonlinear time series. According to this approach, given the embedding dimension $\mathrm{m}$ and the time delay $\mathrm{T}$, for a scalar time series $\mathrm{X}_{\mathrm{i}}$ where $\mathrm{i}=1,2, \ldots, \mathrm{N}$, the dynamics can be fully embedded in $\mathrm{m}$ dimensional phase space represented by the vector, 


$$
Y_{j}=\left(X_{j}, X_{j+\tau} X_{j+2 \tau}, \ldots, X_{j+(m-1) \tau}\right)
$$

Now, the dynamics can be interpreted in the form of an m-dimensional map $f_{T}$ such that $Y_{j+T}=f_{T}\left(Y_{j}\right)$ where $\mathrm{Y}_{\mathrm{j}}$ and $\mathrm{Y}_{\mathrm{j}+\mathrm{T}}$ are vectors of dimension $\mathrm{m}, \mathrm{Y}_{\mathrm{j}}$ being the state at current time $\mathrm{j}$ and $Y_{j+T}$ being the state at future time $j+T$. The approximation of $f_{T}$ can be done using either a global or a local nonlinear model.

The outcomes of these studies affirm the existence of low-dimensional chaos, thus indicating the possibility of only short-term predictions. Better predictions can be obtained using the chaotic approach since it takes into account the dynamics of the irregular hydrological phenomena from a chaotic deterministic view, thereby reducing the model uncertainty. Also, the dynamic approach employing chaotic theory outperforms the traditional stochastic approach in prediction (Jayawardena and Gurung, 2000). Most of these studies rely only on the low correlation dimension as a measure of the chaotic nature of the time series and as an estimate of embedding dimension. Osborne and Provenzale (1989) claimed that a low correlation dimension can also be observed for a linear stochastic process. Hence, it is advised to assess the chaotic nature and to determine the embedding dimension and delay time by employing a variety of methods (Islam and Sivakumar, 2002; Dhanya and Kumar, 2010). Since different methods will give slightly different embedding dimensions and delay times for a single series, one should opt for an ensemble of predictions with a set of these parameters in order to capture the uncertainty in parameter estimation (Dhanya and Kumar, 2010).

The aim of this paper is to analyse the chaotic behaviour and predictability of a streamflow series employing various techniques. Auto-correlation method is used for preliminary investigation to identify chaos and also to determine the delay time for the phase space reconstruction. Optimum embedding dimension is determined using correlation dimension and false nearest neighbor algorithms.

\section{PREDICTABILITY AND CHAOTIC NATURE}

A variety of techniques have emerged for the identification of chaos which include correlation dimension method (Grassberger and Procaccia, 1983a), false nearest neighbor algorithm (Kennel et al., 1992), nonlinear prediction method (Farmer and Sidorowich, 1987), Lyapunov exponent method (Kantz, 1994), Kolmogorov entropy (Grassberger and Procaccia, 1983b), surrogate data method (Theiler et al., 1992) etc. In this study, correlation dimension, false nearest neighbor method and Lyapunov exponent are employed to analyze the chaotic nature of the time series.

\subsection{Lyapunov Exponent}

One of the basic characteristics of a chaotic system is the unpredictability due to the sensitive dependence on initial conditions. The divergence between the trajectories emerging from very close initial conditions will grow exponentially, hence making the system difficult to predict even after a few time steps. Lyapunov exponent gives the averaged information of divergence of infinitesimally close trajectories and thus the unpredictability of the system. Let $\mathrm{s}_{\mathrm{t} 1}$ and $\mathrm{s}_{\mathrm{t} 2}$ be two points in two trajectories in state space such that the distance between them is $\left\|s_{t 1}-s_{t 2}\right\|=\partial_{0}<<1$. After $\Delta t$ time steps

ahead, the distance $\partial_{\Delta t} \cong\left\|s_{t 1+\Delta t}-s_{t 2+\Delta t}\right\|, \partial_{\Delta t}<<1, \Delta t>>1$ follows an exponential relation with initial separation $\partial_{0}$, i.e., $\partial_{\Delta t} \cong e^{\lambda \Delta t} \partial_{0}$, where $\lambda$ is the Lyapunov exponent (Kantz, 1994). Since the rate of separation is different for various orientations of initial separation vector, the total number of Lyapunov exponents is equal to the number of dimensions of the phase space defined, i.e., a spectrum of exponents will be available. Among them, the highest (global) Lyapunov exponent need only be considered, as it determines the total predictability of the system.

Many algorithms have been developed to calculate the maximal Lyapunov exponent (Wolf et al., 1985; Rosenstein et al., 1993; Kantz, 1994). The exponential divergence is examined here using algorithm introduced by Rosenstein et al. (1993). For calculating the maximum Lyapunov exponent, one has to compute 


$$
S(\Delta t)=\frac{1}{N} \sum_{t_{o}=1}^{N} \ln \left(\frac{1}{\left|\mathrm{U}\left(s_{t_{0}}\right)\right|} \sum_{s_{t} \in \mathrm{U}\left(s_{t_{0}}\right)}\left|s_{t_{0}+\Delta t}-s_{t+\Delta t}\right|\right)
$$

Where $s_{t_{0}}$ are reference points or embedding vectors, $\mathrm{U}\left(s_{t_{0}}\right)$ is the neighborhood of $s_{t_{0}}$ with diameter $\xi$. For a reasonable range of $\xi$ and for all embedding dimensions $\mathrm{m}$ which is larger than some minimum dimension $\mathrm{m}_{0}$, if $S(\Delta t)$ exhibits a linear increase, then its slope can be taken as an estimate of the maximal Lyapunov exponent $\lambda$.

The exponential divergence of the nearby trajectories and hence an unstable orbit (chaos) is indicated by a positive $\lambda$. Negative Lyapunov exponents are characteristic of dissipative or non-conservative systems. Their orbits attract to a stable fixed point or periodic orbit. Zero Lyapunov exponents are exhibited by conservative systems for which the orbit is a neutral fixed point.

\subsection{Correlation Dimension Method}

In correlation dimension method, the correlation integral $C(r)$ is estimated using the GrassbergerProcaccia algorithm (1983) which uses the reconstructed phase space of the time series as given in equation (1). According to this algorithm, for an m-dimensional phase space, the correlation integral $\mathrm{C}(\mathrm{r})$ is given by

$$
C(r)=\lim _{N \rightarrow \infty} \frac{2}{N(N-1)} \sum_{\substack{i, j \\(1 \leq i<j \leq N)}} H\left(r-\left|Y_{i}-Y_{j}\right|\right)
$$

where $\mathrm{H}$ is the Heaviside function, with $H(u)=1$ for $u>0$ and $H(u)=0$ for $u \leq 0$ where $u=\left(r-\left|Y_{i}-Y_{j}\right|\right), r$ is the radius of the sphere centered on $Y_{i}$ or $Y_{j}$ and $\mathrm{N}$ is the number of data. For small values of $r$, the correlation integral holds a power law relation on $r, C(r) \sim r^{d}$ where $\mathrm{d}$ is the correlation dimension of the attractor. The correlation exponent or the dimension, $\mathrm{d}$ can be calculated from the slope of the plot of $\log C(r)$ versus log $r$.

For a chaotic series, the correlation exponent saturates to a constant value on increasing the embedding dimension $m$ and the nearest integer above that saturation value indicates the number of variables necessary to describe the evolution in time. On the other hand, if the correlation exponent increases without reaching a constant value on increase in the embedding dimension, the system under investigation is generally considered as stochastic. This is because, contrary to the low dimensional chaotic systems, stochastic systems acquire large dimensional subsets of the system phase space, leading to an infinite dimension value.

However, the sole presence of finite, non-integer dimension correlation dimension is not sufficient to indicate the presence of a strange attractor. Osborne and Provenzale (1989) opposed the traditional view that stochastic processes lead to a non-convergence of the correction dimension by demonstrating that "colored random noises" characterized by a power law power spectrum exhibit a finite and predictable value of the correlation dimension. While the saturation of correlation dimension in low dimensional dynamic systems is due to the phase correlations, for the above mentioned stochastic systems it is mainly due to the shape of the power spectrum (power law). Hence, it would be worthwhile to repeat the correlation dimension on first numerical derivative and phase randomized signal of the original data, to distinguish low dimensional dynamics and randomness (Provenzale et al., 1992).

In the case of stochastic systems, due to the change in the spectral slope on differentiation, the correlation dimension of the differentiated signal will be much larger than that of the original signal. For low dimensional dynamic systems, correlation dimension will be almost invariant. In the case of phase randomized data, the correlation dimension will be the same as that of the original data, provided the convergence of the dimension is forced only by the shape of the power spectrum and not due to any low-dimensional dynamics. 


\subsection{False Nearest Neighbor Method}

The concept of false nearest neighbor is based on the concept that if the dynamics in phase space can be represented by a smooth vector field, then the neighboring states would be subject to almost the same time evolution (Kantz and Schreiber, 2004). Hence, after a short time into the future, any two close neighboring trajectories emerging from them should still be close neighbors. In the present study, the modified algorithm by Hegger and Kantz (1999) in which the fraction of false nearest neighbors are computed in a probabilistic way has been used. The basic idea is to search for all the data points which are neighbors in a particular embedding dimension $\mathrm{m}$ and which do not remain so, upon increasing the embedding dimension to $\mathrm{m}+1$. Considering a particular data point, determine its nearest neighbor in the $\mathrm{m}^{\text {th }}$ dimension. Compute the ratio of the distances between these two points in the $m+1^{\text {th }}$ and $\mathrm{m}^{\text {th }}$ dimensions. If this ratio is larger than a particular threshold $\mathrm{f}$, then the neighbor is false. When the percentage of false nearest neighbors falls to zero (or a minimum value), the corresponding embedding dimension is considered high enough to represent the dynamics of the series.

\section{DATA USED}

The daily streamflow data at Basantpur station of Mahanadi basin, India for the period June 1972 to May 2004 is considered for the present study. The length of Mahanadi river is $860 \mathrm{~km}$. The drainage basin extends over an area of $0.141 \times 10^{6} \mathrm{~km}^{2}$. Because of its wide basin area and devastating floods, numerous studies have been conducted on Mahanadi streamflow focusing on its prediction, flood forecasting, the impact of climate change on the future flows (Asokan and Dutta, 2008; Maity and Nagesh Kumar, 2008; Maity et al., 2010; Mujumdar and Ghosh, 2008).

The location map of the Basantpur station on Mahanadi basin is shown in figure 1(a). The frequency histogram of the daily streamflow series for the study period is shown in figure $1(b)$. The streamflow is widely varying from 0 to $3.5 \times 10^{4} \mathrm{Mm}^{3}$, with maximum frequency falling in the range of $0-1000 \mathrm{Mm}^{3}$. Major portion of the annual streamflow is received in the monsoon months of July, August and September. The non-monsoonal flows are almost invariant, while the monsoon flows show large deviations from the mean.

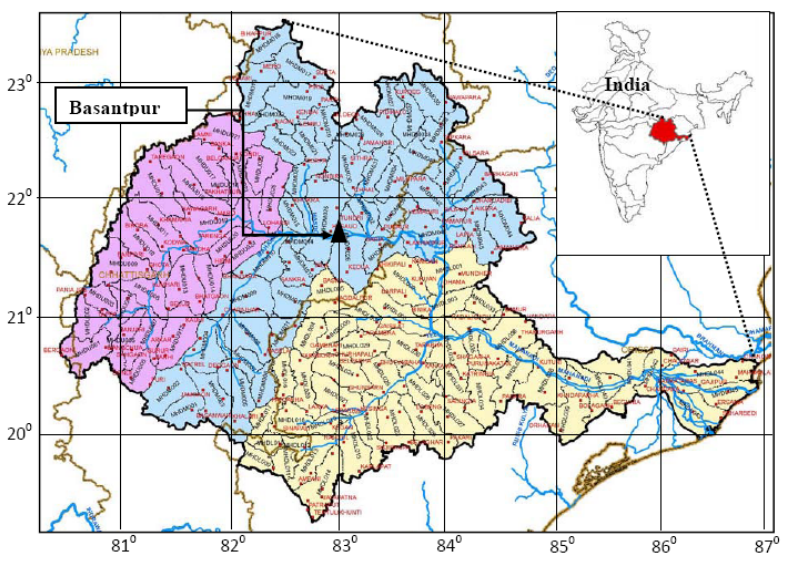

(a)

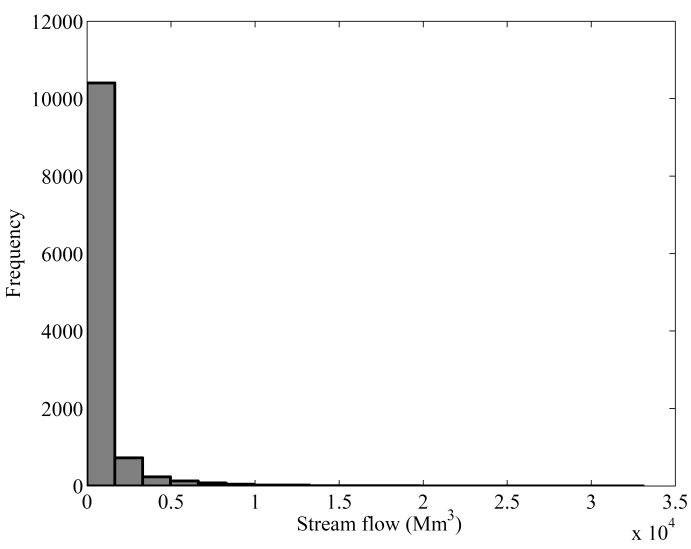

(b)

Figure 1 (a) Location map of the Basantpur station on Mahanadi basin (b) Frequency histogram of Basantpur daily streamflow for the period June 1972 to May 2004

\section{RESULTS AND DISCUSSIONS}

\subsection{Preliminary Investigation of Chaos}

As a preliminary investigation, the autocorrelation function and Fourier spectrum are plotted and are shown in figure 2(a) and 2(b) respectively. The initial exponential decay of autocorrelation functions indicates that the stream flow may be of chaotic nature. The periodic behavior of the autocorrelation function for higher lags is due to the seasonal periodicity. The power spectrum is also exhibiting a broad band form clearly visible for a large frequency range and a power law shape i.e., $P(f) \propto f^{-\alpha}$ with $\alpha \approx 1.35$. The choice of the delay time $\tau$ is made using the autocorrelation method and the mutual information method. In autocorrelation method, the lag time at which the autocorrelation function attains a zero value (fig. $2(a)$ ) i.e., $74^{\text {th }}$ day is considered as the delay time. 


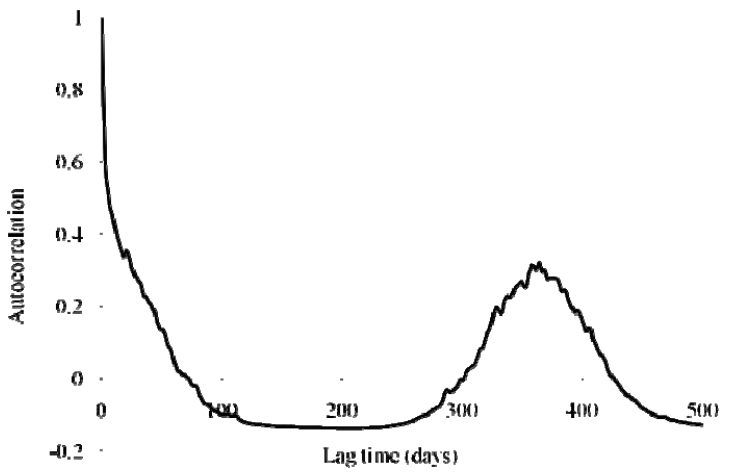

(a)

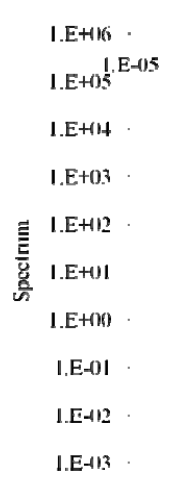

I.E-11,

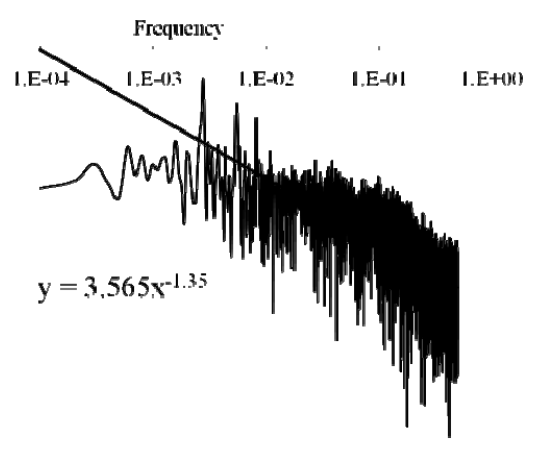

(b)

Figure 2 (a) Autocorrelation function of Basantpur stream flow; (b) Fourier spectrum of Basantpur stream flow

\subsection{Determination of Predictability: Lyapunov Exponent}

Lyapunov exponent provides a measure of the exponential growth due to infinitesimal perturbations. The maximal Lyapunov exponent is calculated employing the algorithm by Rosenstein et al. (1993) which is based on the nearest neighbor approach. The variation of $S(\Delta t)$ with time, $\mathrm{t}$ for Basantpur station at dimensions $m=4$ to 6 is shown in figure 3 . The slope of the linear part of the curve gives the maximum Lyapunov exponent. A positive slope of around 0.167 confirms the exponential divergence of trajectories and hence the chaotic nature of the daily stream flow. The inverse of the Lyapunov exponent defines the predictability of the system, which is around 7 days.

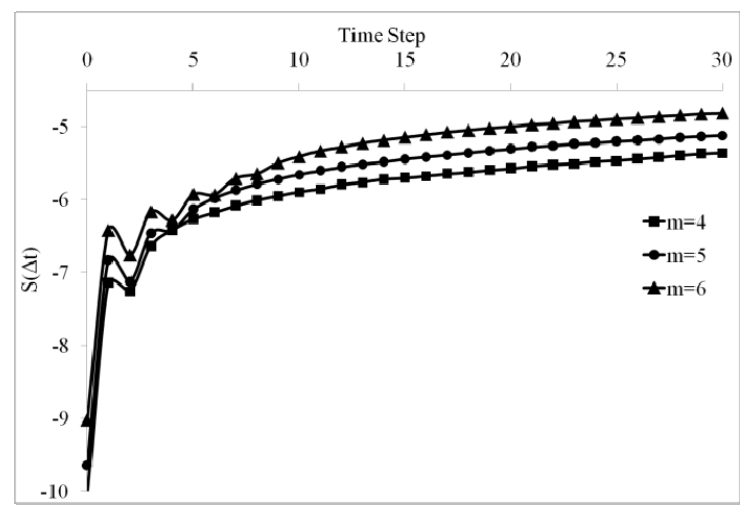

Figure 3 Variation of $S(\Delta t)$ with time for various embedding dimensions

\subsection{Determination of Embedding Dimension}

\subsubsection{Correlation Dimension Method:}

The correlation integral $C(r)$ is calculated according to Grassberger-Procaccia algorithm for embedding dimensions 1 to 40 . A plot of correlation integral $C(r)$ vs. radius $r$ on a log-log scale for embedding dimensions $m=1$ to 40 is shown in figure 4(a). For each of the embedding dimensions, slope of $C(r)$ vs. $r$ over the clear scaling region gives the corresponding correlation exponent. The variation of the correlation exponent with the embedding dimension is shown in figure 4(b). The correlation exponent is increasing with embedding dimension and reaching a constant saturation value at embedding dimension $m \geq 18$, which is an indication of the existence of chaos in the stream flow series. The saturation value is slightly different for different regions. The saturation value of 5.21 at an embedding dimension $m=18$ indicates that the number of variables dominantly influencing the stream flow dynamics is $\approx 6$. The low correlation dimension also suggests the possible presence of lowdimensional chaotic behavior.

The power spectrum of the Basantpur stream flow series is showing a power law behavior with $\alpha \approx 1.35$ as shown in figure 2(b). Since the convergence of the correlation dimension can also be exhibited by some stochastic series due to its power law behavior of power spectrum, it is recommended to perform the correlation dimension method on the first derivative and the phase 
randomized data of the original signal. A comparison of the variations of correlation exponent with embedding dimension for the first derivative of data, phase randomized data and original data are shown in figure 4(c). While the variation of correlation exponent of first derivative is almost identical to that of the original data with almost the same saturation value, the correlation dimension of the phase randomized data set is not converging at all. This eliminates the possibility of linear correlations forcing the saturation of correlation exponent and thereby confirms the presence of a low dimensional strange attractor in the stream flow series.

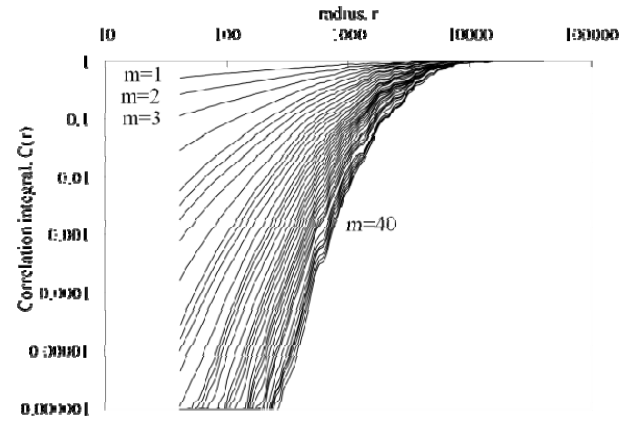

(a)

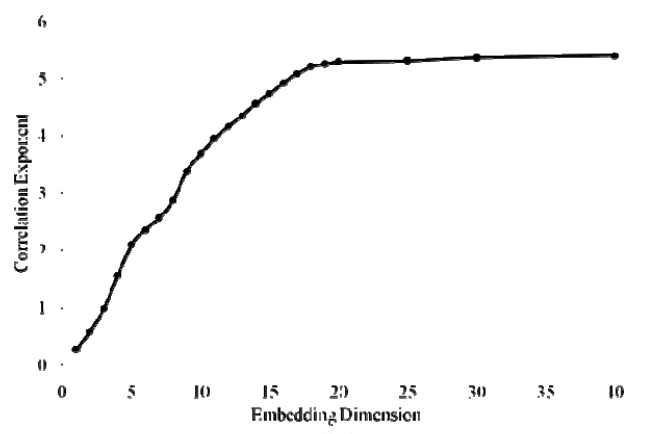

(b)

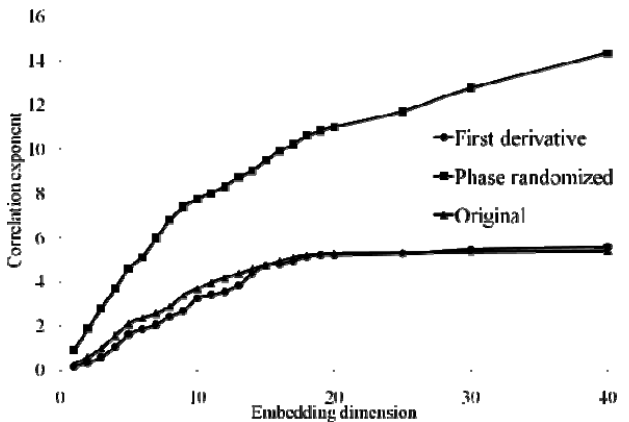

(c)

Figure 4. (a) Variation of correlation integral with radius on a log- log scale for embedding dimensions from 1 to 40; (b) Variation of correlation exponent with embedding dimension; (c) Variation of correlation exponent with embedding dimension for original data, phase randomized data and first derivative of data

\subsubsection{False Nearest Neighbour Method:}

The modified FNN algorithm by Hegger and Kantz (1999) is applied on the stream flow series. The threshold value $f$ is fixed at 5 . The variation of the fraction of false nearest neighbors for different embedding dimensions is shown in figure 5 . The fraction of nearest neighbors is falling to a minimum value at an embedding dimension of 7 , indicating that minimum 7 variables are necessary to explain the entire system. This is in close agreement with the value obtained by the correlation dimension method.

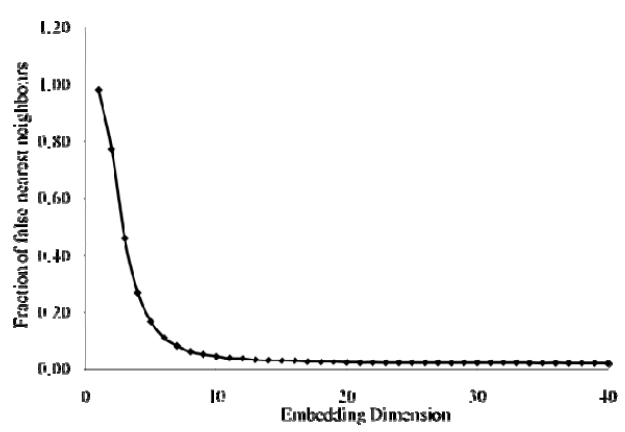

Figure 5. Variation of fraction of false nearest neighbors with embedding dimension

\section{CONCLUSIONS}

The recent interest in nonlinear dynamics and also chaos theory has drawn attention towards considering streamflow as a chaotic system which is much sensitive to initial conditions and short term predictability. The present study was aimed at analyzing the chaotic nature of streamflow series using 
different techniques. The daily streamflow data at Basantpur station of Mahanadi basin, India for the period June 1972 to May 2004.

The positive Lyapunov exponents of the three regions confirm the unpredictability of the systems. The predictability of daily streamflow series is limited to only 7 days. The behaviour of streamflow dynamics was investigated using correlation dimension method with Grassberger-Procaccia algorithm (GPA). The clear scaling region in the $C(r)$ vs. $r$ plot on a log - log scale and also attaining a correlation exponent saturation value of 5.21 indicate a low dimensional chaotic behaviour of the streamflow series.

Since colored random noises also exhibit a finite correlation dimension value, the above method is repeated on phase randomized data and on first derivative of the streamflow series. The correlation dimensions of phase randomized data are not converging, while those of first derivative are almost same as of the original data. This elucidates that the saturation of correlation dimension is not due to the inherent linear correlation in the data; but because of the low dimensional chaotic dynamics present in the data. Since one should not confirm the chaotic nature based on the correlation dimension method alone, false nearest neighbor method is also employed to determine the optimum embedding dimension. The fraction of false nearest neighbours is falling to a minimum value at an embedding dimension of 7 , which indicates that the optimum embedding dimension of the streamflow series is 7 . These results suggest that the seemingly irregular behavior of streamflow process can be better explained though a chaotic framework than through a stochastic representation.

\section{REFERENCES}

Asokan, S.M., and Dutta, D. (2008), Analysis of water resources in the Mahanadi river basin, India under projected climate conditions, Hydrological Processes, 22, $3589-3603$.

Dhanya, C.T. and Kumar, D.N. (2010), Nonlinear ensemble prediction of chaotic daily rainfall, Advances in water resources, 33, 327-347.

Elshorbagy, A., Simonovic, S.P. and Panu, U.S. (2002), Noise reduction in chaotic hydrologic time series: facts and doubts, J. Hydrol., 256(3/4), 845-848.

Farmer, J.D. and Sidorowich, J.J. (1987), Predicting chaotic time series, Phys. Rev. Lett., 59, 845848.

Grassberger, P. and Procaccia, I. (1983a), Measuring the strangeness of strange attractors, Physica D, 9, 189-208.

Grassberger, P. and Procaccia, I. (1983b), Estimation of the Kolmogorov entropy from a chaotic signal, Phys. Rev. A, 28, 2591-2593.

Hegger, R. and Kantz, H. (1999), Improved false nearest neighbor method to detect determinism in time series data, Phys. Rev. E, 60, $4970-4973$.

Islam, M.N. and Sivakumar, B. (2002), Characterization and prediction of runoff dynamics: a nonlinear dynamical view, Adv. Wat. Resour., 25, 179-190.

Jayawardena, A.W. and Gurung, A.B. (2000), Noise reduction and prediction of hydrometeorological time series: dynamical systems approach vs. stochastic approach, J. Hydrol., 228, 242-264.

Jayawardena, A.W. and Lai, F. (1994), Analysis and prediction of chaos in rainfall and stream flow time series, J. Hydrol., 153, 23-52.

Kantz, H. (1994), A robust method to estimate the maximal Lyapunov exponent of a time series, Phys. Lett. A, 185, 77-87.

Kantz, H. and Schreiber, T. (2004), Nonlinear Time Series Analysis, $2^{\text {nd }}$ ed., Cambridge University Press, Cambridge, UK.

Kennel, M.B., Brown, R. and Abarbanel, H.D.I. (1992), Determining embedding dimension for phase space reconstruction using a geometric method, Phys. Rev. A, 45, 3403-3411.

Liu, Q., Islam, S., Rodriguez-lturbe, I. and Le, Y. (1998), Phase-space analysis of daily streamflow: characterization and prediction, Adv. Wat. Resour., 21, 463-475.

Lorenz, E.N. (1972), Predictability: Does the flap of a Butterfly's wings in Brazil set off a Tornado in Texas?, AAAS section on environmental Sciences New Approached to Global weather: GARP (The Global Atmospheric research Program), $139^{\text {th }}$ meeting, American Association for the Advancement of Science.

Maity, R. and Nagesh Kumar, D. (2008), Basin-scale streamflow forecasting using the information of large-scale atmospheric circulation phenomena, Hydrological Processes, 22, 643 - 650.

Maity, R., Bhagwat, P.P. and Bhatnagar, A. (2010), Potential of spport vector regression for prediction of monthly streamflow using endogenous property, Hydrological Processes, 24, 917 - 923.

Mujumdar, P.P., and Ghosh, S. (2008), Modeling GCM and scenario uncertainty using a possibilistic approach: Application to the Mahanadi River, India, Water Resour. Res., 44, W06407.

Osborne, A.R. and, Provenzale, A. (1989), Finite correlation dimension for stochastic systems with 
power law spectra, Physica D., 35, 357-381.

Porporato, A. and Ridolfi, L. (1996), Clues to the existence of deterministic chaos in river flow, Int. J. Mod. Phys. B., 10(15), 1821-1862.

Porporato, A. and Ridolfi, L. (1997), Nonlinear analysis of river flow time sequences, Water Resour. Res., 33(6), 1353-1367.

Provenzale, A., Smith, L.A., Vio, R. and Murante, G. (1992), Distinguishing between low-dimensional dynamics and randomness in measured time series, Physica $D, 58,31-49$.

Puente, C.E. and Obregon, N. (1996), A deterministic geometric representation of temporal rainfall: results for a storm in Boston, Water Resour. Res., 32(9), 2825-2839.

Rodriguez-Iturbe, I.., De Power, F.B., Sharifi, M.B. and Georgakakos, K.P. (1989), Chaos in rainfall, Water Resour. Res., 25(7), 1667-1675.

Rosenstein, M. T., Collins, J. J. and De Luca, C. J. (1993), A practical method for calculating largest Lyapunov exponents from small data sets, Physica D, 65, 117-134.

Sangoyomi, T., Lall, U., and Abarbanel, H.D.J. (1996), Nonlinear dynamics of the Great Salt Lake: dimension estimation, Water Resour. Res., 32(1), 149-159.

Shang, P., Xu, N. and Kamae, S. (2009), Chaotic analysis of time series in the sediment transport phenomenon, Chaos, Solitons \& Fractals, 41(1), 368-379.

Sivakumar, B. (2001), Rainfall dynamics at different temporal scales: A chaotic perspective, Hydrol. Earth System Sci., 5(4), 645-651.

Sivakumar, B., Berndtsson, R., Olsson, J. and Jinn, K. (2001), Evidence of chaos in the rainfall-runoff process, Hydrol. Sci. J., 46(1), 131-145.

Sivakumar, B., Liong, S.Y., Liaw, C.Y. and Phoon, K.K. (1999), Singapore rainfall behavior: chaotic?, J. Hydrol. Eng., 4(1), 38-48.

Smith, L.A., Ziehmann, C. And Fraedrich, K. (1998), Uncertainty dynamics and predictability in chaotic systems, Q.J.R. Meteorol. Soc. 125, 2855-2886.

Takens, F. (1981), Detecting strange attractors in turbulence, in: D.A. Rand, L.S. Young (Eds.) Lectures Notes in Mathematics, 898, Springer-Verlag,Berlin, Germany.

Wang, Q. and Gan, T.Y. (1998), Biases of correlation dimension estimates of streamflow data in the Canadian prairies, Water Resour. Res., 34(9), 2329-2339.

Wolf, A., Swift, J.B., Swinney, H.L. and Vastano, A. (1985), Determining Lyapunov exponents from a time series, Physica D, 16, 285-317. 